\title{
Diabetes increases the mortality of patients with COVID-19: a meta-analysis
}

\author{
Zeng-hong Wu ${ }^{1} \cdot$ Yun Tang ${ }^{2} \cdot$ Qing Cheng ${ }^{3}$
}

Received: 23 April 2020 / Accepted: 6 May 2020 / Published online: 24 June 2020

(c) Springer-Verlag Italia S.r.l., part of Springer Nature 2020

\begin{abstract}
Aims Nowadays, the ongoing pandemic of COVID-19 caused by the novel coronavirus Syndrome-Coronavirus-2 (SARS$\mathrm{CoV}-2$ ) is an emerging, rapidly evolving situation. Complications such as hypertension, diabetes, COPD, cardiovascular disease, and cerebrovascular disease are major risk factors for patients with COVID-19.

Methods No meta-analysis has explored if or not diabetes related to mortality of patients with COVID-19. Therefore, this meta-analysis first aims to explore the possible clinical mortality between diabetes and COVID-19, analyze if diabetes patients infected with SARS-CoV-2 are exposed to the worst clinical prognostic risk, and to evaluate the reliability of the evidence. Results Our results showed a close relationship between diabetes and mortality of COVID-19, with a pooled OR of 1.75 (95\% CI 1.31-2.36; $P=0.0002)$. The pooled data were calculated with the fixed effects model (FEM) as no heterogeneity appeared in the studies. Sensitivity analysis showed that after omitting any single study or converting a random effect model to FEM, the main results still held.

Conclusions Our meta-analysis showed that diabetes increases the mortality of patients with COVID-19. These results indicated the disturbance of blood glucose in the COVID-19 patients. More importantly, this meta-analysis grades the reliability of evidence for further basic and clinical research into the diabetes dysfunction in COVID-19 patients.
\end{abstract}

Keywords COVID-19 $\cdot$ SARS-CoV-2 $\cdot$ Diabetes $\cdot$ Meta-analysis

\section{Abbreviations \\ SARS-CoV-2 Syndrome-Coronavirus-2 \\ $\mathrm{COV} \quad$ Coronaviruses $(\mathrm{CoV})$ \\ ACE2 Angiotensin converting enzyme 2}

Managed by Antonio Secchi.

\section{Qing Cheng}

cqij74@163.com

1 Department of Infectious Diseases, Union Hospital, Tongji Medical College, Huazhong University of Science and Technology, Wuhan 430022, China

2 Department of Critical Care Medicine, Union Hospital, Tongji Medical College, Huazhong University of Science and Technology, Wuhan 430022, China

3 Department of Otorhinolaryngology, Union Hospital, Tongji Medical College, Huazhong University of Science and Technology, Wuhan, Hubei, China

\section{Introduction}

Coronaviruses (CoVs) are enveloped viruses with a positive single-stranded RNA virus, which are widely distributed in humans and animals to cause respiratory infections in humans [1]. Nowadays, the ongoing pandemic of COVID-19 caused by the novel coronavirus Syndrome-Coronavirus- 2 (SARS-CoV-2) is an emerging, rapidly evolving situation [2]. As of April 14, 2020, 1,924,626 cases and 119,625 deaths in total have been confirmed around the world, suggesting that the overall death rate of COVID-19 was $6.2 \%$. Although SARS-CoV-2 and SARS-CoV share similarities in phylogeny and clinical trials, the new type of coronavirus seems to have a higher spread and lower mortality [3]. A study reported that SARS-CoV-2 invades into the cells through its predicated receptor angiotensin converting enzyme 2 (ACE2) [4]. Blocking the renin-angiotensin system can prevent new-onset diabetes and reduce the risk of diabetes complications [5]. Du et al. [6] found that the elderly who are over 65 years old showed higher risk of suffering from the disease, especially those with severe complications and patients who had experience of entering 
the intensive care unit (ICU) than those who did not. This finding indicated that complications may be a risk factor for adverse consequences. A recent meta-analysis reported that hypertension, diabetes, COPD, cardiovascular disease, and cerebrovascular disease are major risk factors for patients with COVID-19, but the impact of these risk factors on COVID-19 mortality has not been explored [7]. Therefore, this meta-analysis first aims to explore the possible clinical mortality between diabetes and COVID-19, analyze if diabetes patients infected with SARS-CoV-2 are exposed to the worst clinical prognostic risk, and to evaluate the reliability of the evidence. This result may help the government formulate prevention policies and respond to the outbreak of COVID-19, ultimately contributing to the better clinical management of patients with COVID-19 and diabetes.

\section{Methods}

\section{Search strategy}

The preferred reporting items for meta-analyses (PRISMA) guidelines were used. We determined to include studies involving in COVID-19, SARS-CoV-2, or diabetes. To find relevant primitive articles, we performed a comprehensive retrieval in databases, involving Medline via PubMed, EMBASE, and Web of Science with the following words: 'COVID-19,' 'SARS-CoV-2,' 'Wuhan virus,' 'Chinese virus,' 'Novel coronavirus,' 'Novel coronavirus 2019,' '2019 nCoV,' 'Wuhan coronavirus,' 'Wuhan pneumonia,' 'The 2019 coronavirus,' 'died,' 'non-survivors,' 'death,' 'Survival,' 'survivors,' and 'alive.' Papers were searched in the databases until April 14, 2020, with no restrictions of language. We also reviewed reference lists based on the recognized literature to find additional eligible studies. We first screened both titles and abstracts of the articles, and publications which may involve in data concerning COVID19, SARS-CoV-2, or diabetes.

\section{Inclusion and exclusion standard, data extraction}

Primitive studies were carefully examined, and there were no nation restrictions in our study. The inclusion criteria were as follows: (1) all studies reporting data on COVID-19 nonsurvivors, survivors, and laboratory-confirmed COVID-19 patients; (2) studies limited to mankind, contained original data, published in English and appeared in the form of either abstract or full-text. The exclusion criteria were as follows: (1) duplicate studies, letters, case reports, abstracts, or reviews; (2) research focusing on morbidity rather than mortality. We collected the following information from each study: publication year, first author's name, sample size, age for both dead group, and alive group.

\section{Statistical analysis}

We assessed the quality of each involved research by the Newcastle-Ottawa Scale [8]. Meta-analysis was conducted via Review Manager 5.3. By calculating the $I^{2}$ index to evaluate the heterogeneity, the $I^{2}$ values of $<25 \%, 25-50 \%, 50-75 \%$, and $75-100 \%$ were considered as homogeneous, low, moderate, and high heterogeneous levels, respectively. If the $I^{2}$ value was $>50 \%$, the random effects model (REM) would be applied. If the $I^{2}$ value was $<50 \%$, the fixed effects model (FEM) would be applied. The pooled odds ratios (ORs) of different studies and corresponding $95 \%$ confidence intervals (CIs) were used to estimate the relationship between diabetes and the mortality of patients with COVID-19. Then, the sensitivity analysis was repeated, with the impact of each study evaluated by deleting different individual studies each time.

\section{Results}

\section{Study processing}

A total of 597 potentially relevant articles were identified using our search strategy from databases and 1 from reference lists. Four hundred and sixty studies remained after ruling out duplicates. After screening the titles and abstracts, 347 research articles that did not satisfy the inclusion criteria were ruled out. After scanning the main body of the reserving 13 records, another seven articles were excluded. Finally, nine qualified articles met the eligibility criteria [9-17]. The steps of document retrieval are shown in Fig. 1. Meanwhile, the common characteristic of included studies is listed in Table 1 . The metaanalysis involved 926 cases (260 dead and 666 alive) overall that were all published in 2020. One study [9] reported the settings in ICU and isolation wards. All articles are of high quality because NOS score was no less than 6 .

\section{Pooled analysis}

The outcome of the forest plot for the connection between diabetes and the mortality of patients with COVID-19 is shown in Fig. 2. Our results showed a close relationship between diabetes and mortality of COVID-19, with a pooled OR of 1.75 (95\% CI 1.31-2.36; $P=0.0002$ ). The pooled data were calculated with the FEM as no heterogeneity appeared in the studies.

\section{Subgroup analysis and sensitivity analysis}

We chose age as the subgroup, and there was no significant difference between age $\geq 70$ subgroup and age $<70$ subgroup, and the OR was 1.33 (95\% CI $0.78-2.28, P=0.30)$ and 2.05 (95\% CI 1.44-2.94, $P<0.001$ ), respectively, in Fig. 3. As a result 


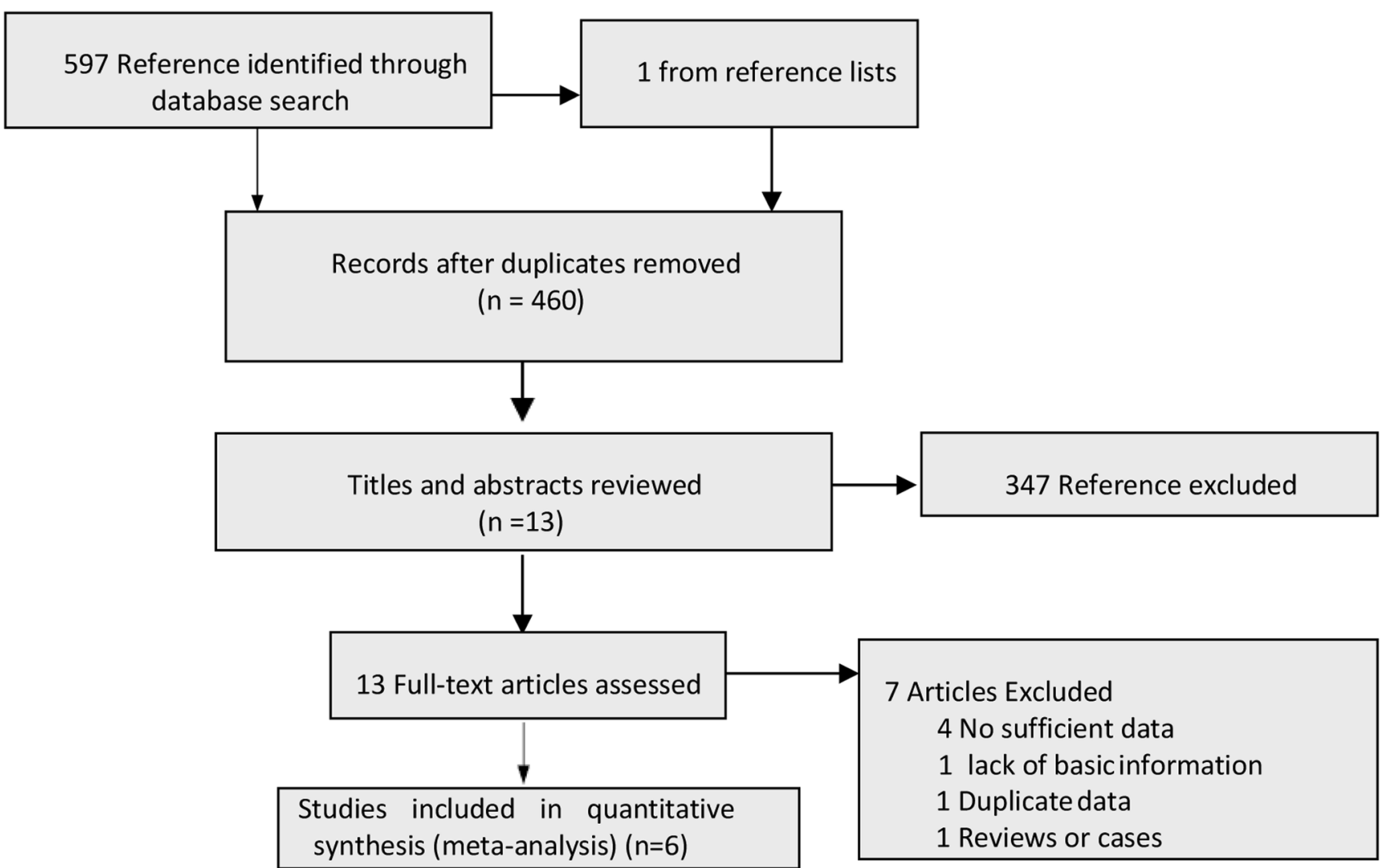

Fig. 1 Search strategy to identify articles on the relationship between diabetes and the mortality of patients with COVID-19

Table 1 Description of included studies

\begin{tabular}{|c|c|c|c|c|c|c|}
\hline Study & Setting & Country & Sample (dead/alive) & Age (dead/alive) & Female & NOS \\
\hline Yang et al. [9] & ICU & China & $32 / 20$ & $64.6(11.2) / 51.9(12.9)$ & $11 / 6$ & 7 \\
\hline Wang et al. [10] & Isolation ward & China & $65 / 274$ & $76(70-83) / 68(64-74)$ & $26 / 147$ & 7 \\
\hline Chen et al. [11] & Isolation ward & China & $113 / 161$ & $68.0(62.0-77.0) / 51.0(37.0-66.0)$ & $30 / 73$ & 8 \\
\hline Chen et al. [12] & Isolation ward & China & $19 / 36$ & $77 / 72$ & $3 / 18$ & 7 \\
\hline Yuan et al. [13] & Isolation ward & China & $10 / 17$ & $68(63-73 / 55(35-60)$ & $6 / 9$ & 7 \\
\hline Ruan et al. [15] & Isolation ward & China & $68 / 82$ & $67(15-81) / 50(44-81)$ & $19 / 29$ & 7 \\
\hline Zhou et al. [16] & Isolation ward & China & $54 / 137$ & $69.0(63.0-76.0) / 52.0(4.0-58.0)$ & $16 / 56$ & 7 \\
\hline Deng et al. [17] & Isolation ward & China & $17 / 116$ & $69(62-74) / 40(33-57)$ & $36 / 65$ & 7 \\
\hline Du et al. [14] & Isolation ward & China & $21 / 158$ & $70.2(7.7) / 56.0(13.5)$ & $11 / 71$ & 7 \\
\hline
\end{tabular}

Data expressed by mean (SD) or median (IQR)

$I Q R$ interquartile range, $I C U$ intensive care unit

of insufficient data, the impact of age stratification on mortality of COVID-19 should be further carried out. Meanwhile, our study demonstrated no heterogeneity $\left(I^{2}=5 \%\right)$, and upon eliminating the impact of Yuan's study and Wang's study, the results significantly affected the pooled outcomes $\left(I^{2}\right.$ dropped from $5 \%$ to 0 ). Therefore, we assumed that the studies of Yuan et al. and Wang et al. may be the sources of heterogeneity in this meta-analysis. The sensitivity analysis results showed that the ORs ranged from 1.50 (95\% CI 1.01-2.23) to 2.03 (95\%
CI 1.29-3.20). The risk of publication bias was analyzed by Begg's test $(P=0.09)$ and Egger's regression test $(P=0.36)$, indicating no significant publication bias in our meta-analysis. 


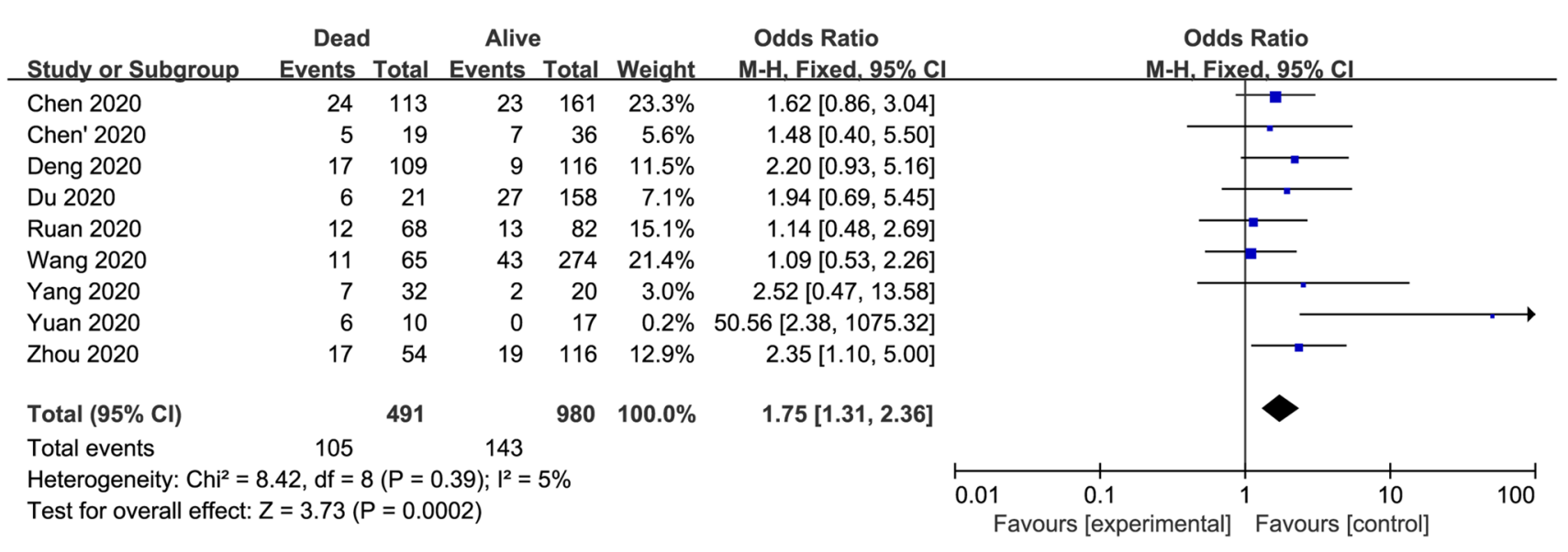

Fig. 2 Forest plot of the relationship between diabetes and the mortality of patients with COVID-19

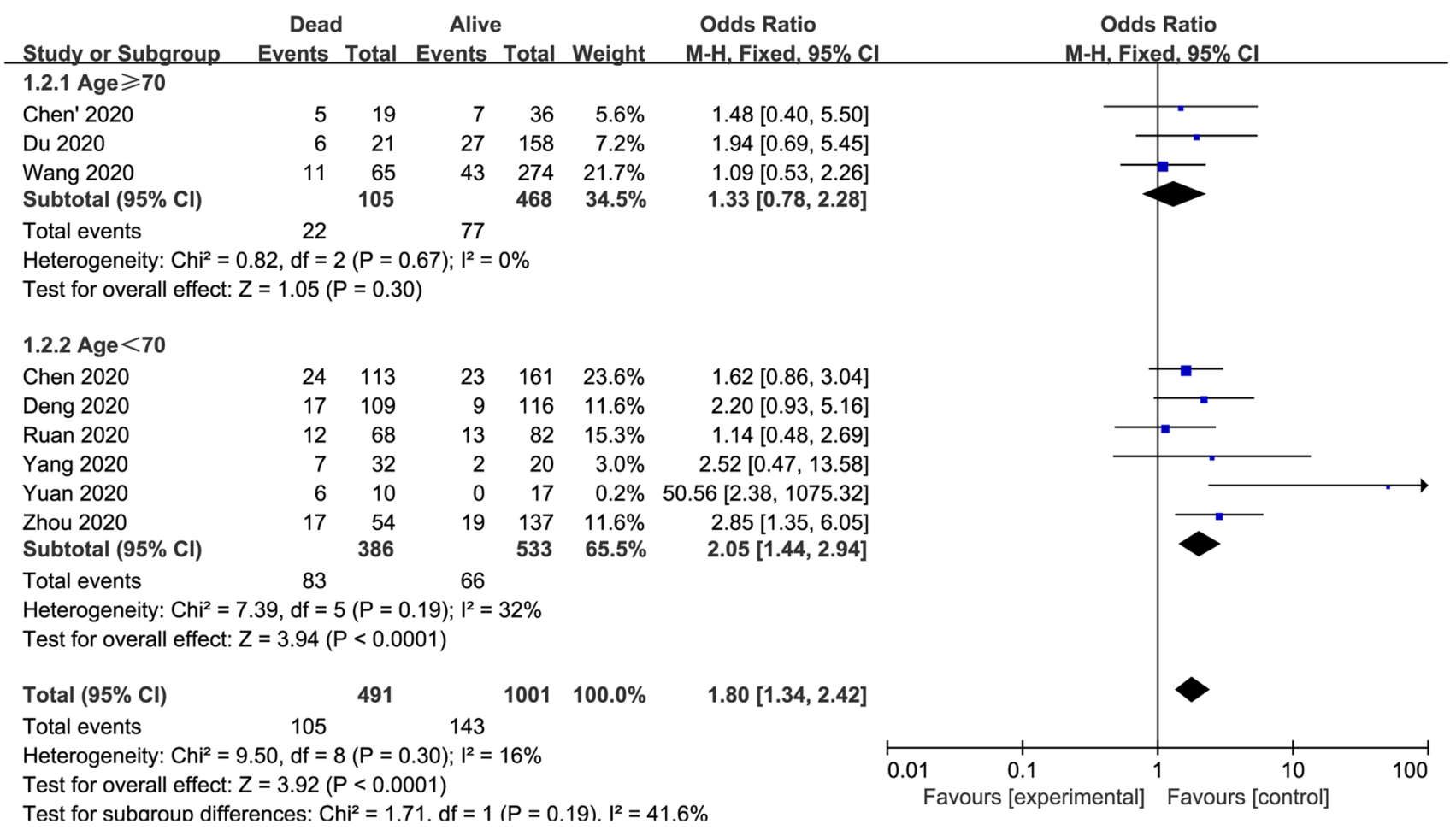

Fig. 3 Forest plot of age subgroup analysis

\section{Discussion}

Our meta-analysis suggested that the mortality of patients with COVID-19 was significantly related to diabetes with OR of 1.75 (95\% CI 1.31-2.36; $P=0.0002)$. Meanwhile, the value of $I^{2}=5 \%\left(I^{2}<50, P<0.01\right)$ indicated that no heterogeneity exists in the studies. In addition, sensitivity analysis revealed that when excluding any single study or REM was converted to FEM, the total outcomes still held.
So, we are confident in the validity of the data obtained in our study, showing a powerful correlation between diabetes and the mortality of patients with COVID-19.

Despite the unclear pathophysiology, it has been observed that the most severe and fatal cases of COVID-19 occur in the elderly or patients with potential complications, especially those with diabetes [18]. According to the reports, diabetes and high blood glucose levels are important predictors of the severity and death of patients infected with different viruses, involving SARS-CoV and MERS-CoV [19, 20]. A 
study found elderly patients with chronic diseases, including diabetes, were at higher risk of severe COVID-19 infection and death [21]. Insulin resistance promotes synthesis of end-glycosylation products and stimulates the production of pro-inflammatory cytokines, oxidative stress, and adhesion molecules, resulting in a higher likelihood of infection and worse prognosis [22].

Infection leads to destruction of pancreatic beta cells, decreased pancreatic insulin content, and changes in the host's ability to respond normally to glucose tolerance tests [23]. A previous study suggested that SARS coronavirus uses ACE2 as a receptor to enter and damage islet cells that cause acute diabetes [24]. Until now, there was no relevant study exploring the role of ACE2 in COVID-19 diabetic patients. A recent meta-analysis retrieved the data of 2108 Chinese patients diagnosed with SARS-Cov-2 infection and found the prevalence of diabetes is $10.3 \%$ [25]. But, until now, no meta-analysis has explored the possible mortality between diabetes and COVID-19. Therefore, our meta-analysis results found a significant relationship between diabetes and mortality COVID-19, with a pooled OR of 1.70 (95\% CI $1.16-2.48 ; P=0.006$ ), which may contribute to better clinical management of patients with COVID-19 and diabetes.

Compared with younger patients, patients aged 65 and over showed higher risk of initial complications, more severe symptoms, and were more prone to multiple organ involvement and death [12]. Similarly, Feng et al. [26] recruited 476 COVID-19 patients from three different cities in China and the survival rate of patients over 75 years old was significantly lower than that of young patients. Factors leading to poor health include physiological changes in aging and multiple age-related complications. A study held the opinion that older people's suspicion and detection threshold for SARS-2 should be lower, such as temperature, the decline in pulmonary function, and shortness of breath [27]. Management began with the diagnosis and classification of the elderly in order to achieve an appropriate level of care. Simultaneously, our age subgroup analysis results indicated that there was no significant difference between age $\geq 70$ subgroup and age $<70$ subgroup. However, if we chose the threshold for age stratification as 65 years old, in the age $\geq 65$ subgroup, the OR would be 1.73 (95\% CI $1.28-2.34 ; P=0.0003)$. So, more research on the impact of age stratification on mortality of COVID-19 diabetic patients should be carried out.

This is the first attempt to use meta-analysis to prove whether the mortality of patients with COVID-19 is related to diabetes. At the same time, our study has some limitations. First, due to the inadequate data, we do not have access to information such as diabetes duration, time of diagnoses, diabetes type, diabetes medications and complications, and thus we cannot conduct risk stratification in the subgroup analysis within the diabetic population. Second, we only obtained the information regarding the age and gender of COVID-19 patients, but other factors might influence the accuracy of results, such as BMI [28], measurements, and instruments for the detection of SARS-CoV-2. Meanwhile, the populations included in our study were mainly in Asian Populations and so these conclusions, even if important, cannot be extended automatically to Caucasian or other populations. Finally, the patients who are diagnosed with COVID-19 may have multiple chronic diseases simultaneously, such as hypertension, cardiovascular disease, cerebrovascular disease, and COPD, which may affect the accuracy of the results. Meanwhile, the samples are not quite enough and more large-scale studies are needed.

\section{Conclusion}

Our meta-analysis showed that diabetes increases the mortality of patients with COVID-19. These results indicated the disturbance of blood glucose in the COVID-19 patients. More importantly, this meta-analysis grades the reliability of evidence for further basic and clinical research into the diabetes dysfunction in COVID-19 patients.

Author contributions WZH and TY designed and analyzed the research study; WZH and CQ wrote and revised the manuscript, WZH collected the data, and all authors have read and approved the manuscript.

Funding This work is not supported by Grants.

\section{Compliance with ethical standards}

Conflict of interest All authors certify that they have no affiliations with or involvement in any organization or entity with any financial interest (such as honoraria; educational grants; participation in speakers' bureaus; membership, employment, consultancies, stock ownership, or other equity interest; and expert testimony or patent-licensing arrangements), or non-financial interest (such as personal or professional relationships, affiliations, knowledge or beliefs) in the subject matter or materials discussed in this manuscript.

Ethical standards Not applicable.

Informed consent For this type of study, informed consent is not required.

\section{References}

1. Liu Y, Yang Y, Zhang C et al (2020) Clinical and biochemical indexes from 2019-nCoV infected patients linked to viral loads and lung injury. Sci China Life Sci 63:364-374

2. Gorbalenya AE, Baker SC, Baric RS et al (2020) The species severe acute respiratory syndromerelated coronavirus: classifying 2019-nCoV and naming it SARS-CoV-2. Nat Microbiol 5(4):536544. https://doi.org/10.1038/s41564-020-0695-z 
3. Ceccarelli M, Berretta M, Venanzi Rullo E, Nunnari G, Cacopardo B (2020) Differences and similarities between severe acute respiratory syndrome (SARS)-coronavirus $(\mathrm{CoV})$ and SARS-CoV-2. Would a rose by another name smell as sweet? Eur Rev Med Pharmacol Sci 24(5):2781-2783. https://doi.org/10.26355/eurre v_202003_20551

4. Walls AC, Park Y-J, Tortorici MA, Wall A, McGuire AT, Veesler D (2020) Structure, function, and antigenicity of the SARS-CoV-2 spike glycoprotein. Cell. https://doi.org/10.1016/j. cell.2020.02.058

5. Bindom SM, Lazartigues E (2008) The sweeter side of ACE2: physiological evidence for a role in diabetes. Mol Cell Endocrinol 302(2): 193-202

6. Du RH, Liu LM, Yin W et al (2020) Hospitalization and critical care of 109 decedents with COVID-19 Pneumonia in Wuhan, China. Ann Am Thorac Soc. https://doi.org/10.1513/Annal sATS.202003-225OC

7. Wang B, Li R, Lu Z et al (2020) Does comorbidity increase the risk of patients with COVID-19: evidence from meta-analysis. Aging (Albany NY). https://doi.org/10.18632/aging.103000

8. Stan A (2010) Critical evaluation of the Newcastle-Ottawa scale for the assessment of the quality of nonrandomized studies in meta-analyses. Eur J Epidemiol 25(9):603-605. https://doi. org/10.1007/s10654-010-9491-z

9. Yang X, Yu Y, Xu J et al (2020) Clinical course and outcomes of critically ill patients with SARS-CoV-2 pneumonia in Wuhan, China: a single-centered, retrospective, observational study. Lancet Respir Med. https://doi.org/10.1016/S2213-2600(20)30079-5

10. Wang L, He W, Yu X et al (2019) Coronavirus disease 2019 in elderly patients: characteristics and prognostic factors based on 4-week follow-up. J Infect. https://doi.org/10.1016/j. jinf.2020.03.019

11. Chen T, Wu D, Chen $\mathrm{H}$ et al (2020) Clinical characteristics of 113 deceased patients with coronavirus disease 2019: retrospective study. BMJ 368:m1091. https://doi.org/10.1136/bmj.m1091

12. Chen TL, Dai Z, Mo P et al (2020) Clinical characteristics and outcomes of older patients with coronavirus disease 2019 (COVID19) in Wuhan, China (2019): a single-centered, retrospective study. J. Gerontol A Biol Sci Med Sci. https://doi.org/10.1093/ gerona/glaa089

13. Yuan M, Yin W, Tao Z et al (2020) Association of radiologic findings with mortality of patients infected with 2019 novel coronavirus in Wuhan, China. PLoS ONE 15:e0230548. https://doi. org/10.1371/journal.pone. 0230548

14. Du R-H, Liang L-R, Yang C-Q et al (2020) Predictors of mortality for patients with COVID-19 pneumonia caused by SARSCoV-2: a prospective cohort study. Eur Respir J. https://doi. org/10.1183/13993003.00524-2020

15. Ruan Q, Yang K, Wang W et al (2020) Correction to: clinical predictors of mortality due to COVID-19 based on an analysis of data of 150 patients from Wuhan, China. Intensive Care Med. https://doi.org/10.1007/s00134-020-06028-Z

16. Zhou F, Yu T, Du R et al (2020) Clinical course and risk factors for mortality of adult inpatients with COVID-19 in Wuhan, China: a retrospective cohort study. Lancet 395(10229):1054-1062. https ://doi.org/10.1016/S0140-6736(20)30566-3

17. Deng Y, Liu W, Liu K et al (2020) Clinical characteristics of fatal and recovered cases of coronavirus disease 2019 (COVID-19) in Wuhan, China: a retrospective study. Chin Med J (Engl). https:// doi.org/10.1097/CM9.0000000000000824

18. Wu Z, McGoogan JM (2020) Characteristics of and important lessons from the coronavirus disease 2019 (COVID-19) outbreak in China: summary of a report of 72314 cases from the Chinese Center for Disease Control and Prevention. JAMA. https://doi. org/10.1001/jama.2020.2648

19. Yang JK, Feng Y, Yuan MY et al (2006) Plasma glucose levels and diabetes are independent predictors for mortality and morbidity in patients with SARS. Diabet Med 23(6):623-628. https://doi.org/1 0.1111/j.1464-5491.2006.01861.x

20. Banik GR, Alqahtani AS, Booy R, Rashid H (2016) Risk factors for severity and mortality in patients with MERS-CoV: analysis of publicly available data from Saudi Arabia. Virol Sin 31(1):81-84. https://doi.org/10.1007/s12250-015-3679-z

21. Ghosh A, Gupta R, Misra A et al (2020) Telemedicine for diabetes care in India during COVID19 pandemic and national lockdown period: guidelines for physicians. Diabetes Metab Syndr 14(4):273-276. https://doi.org/10.1016/j.dsx.2020.04.001

22. Knapp S (2013) Diabetes and infection: is there a link? A minireview. Gerontology 59(2):99-104. https://doi.org/10.1159/00034 5107

23. Onodera T, Jenson AB, Yoon JW et al (1978) Virus-induced diabetes mellitus: reovirus infection of pancreatic $\beta$ cells in mice. Science 201(4355):529-531

24. Yang J-K, Lin S-S, Ji X-J et al (2010) Binding of SARS coronavirus to its receptor damages islets and causes acute diabetes. Acta Diabetol 47:193-199

25. Fadini GP, Morieri ML, Longato E, Avogaro A (2020) Prevalence and impact of diabetes among people infected with SARS-CoV-2. J Endocrinol Investig. https://doi.org/10.1007/s40618-020-01236 $-2$

26. Feng Y, Ling Y, Bai T et al (2020) COVID-19 with different severity: a multi-center study of clinical features. Am J Respir Crit Care Med. https://doi.org/10.1164/rccm.202002-0445OC

27. Nikolich-Zugich J, Knox KS, Rios CT et al (2020) SARS-CoV-2 and COVID-19 in older adults: what we may expect regarding pathogenesis, immune responses, and outcomes. Geroscience. https://doi.org/10.1007/s11357-020-00186-0

28. Luzi L, Radaelli MG (2020) Influenza and obesity: its odd relationship and the lessons for COVID-19 pandemic. Acta Diabetol. https://doi.org/10.1007/s00592-020-01522-8

Publisher's Note Springer Nature remains neutral with regard to jurisdictional claims in published maps and institutional affiliations. 